KYUNGPOOK Math. J. 55(2015), 259-266

http://dx.doi.org/10.5666/KMJ.2015.55.2.259

pISSN 1225-6951 eISSN 0454-8124

(C) Kyungpook Mathematical Journal

\title{
On Graded Quasi-Prime Submodules
}

\author{
Khaldoun Al-Zoubi* \\ nology, P.O. Box 3030, Irbid 22110, Jordan \\ e-mail : kfzoubi@just.edu.jo \\ RASHID ABU-DAWWAS \\ Department of Mathematics, Yarmouk University, Irbid, Jordan \\ e-mail : rrashid@yu.edu.jo
}

Department of Mathematics and Statistics, Jordan University of Science and Tech-

Abstract. Let $G$ be a group with identity $e$. Let $R$ be a $G$-graded commutative ring and $M$ a graded $R$-module. In this paper, we introduce the concept of graded quasi-prime submodules and give some basic results about graded quasi-prime submodules of graded modules. Special attention has been paid, when graded modules are graded multiplication, to find extra properties of these submodules. Furthermore, a topology related to graded quasi-prime submodules is introduced.

\section{Introduction}

Graded prime submodules of graded modules over graded commutative rings have been introduced and studied in $[2,5]$. Here we introduce the concept of graded quasi-prime submodules and we investigate some properties of graded quasi-prime submodules of graded modules over graded commutative rings and consider some conditions under which a graded quasi-prime submodule of a graded module is graded prime. Also, the behavior of graded quasi-prime submodules under localization is studied. Furthermore, we introduce a topology on the set of graded quasi-prime submodules and some properties of this topology are given.

Before we state some results, let us introduce some notation and terminologies. Let $G$ be a group with identity $e$ and $R$ be a commutative ring. Then $R$ is a $G$ graded ring if there exist additive subgroups $R_{g}$ of $R$ such that $R=\bigoplus_{g \in G} R_{g}$ and $R_{g} R_{h} \subseteq R_{g h}$ for all $g, h \in G$. We denote this by $(R, G)$. The elements of $R_{g}$ are called homogeneous of degree $g$ where $R_{g}$ is the additive subgroup of $R$ indexed

* Corresponding Author.

Received November 29, 2013; revised August 23, 2014; accepted October 2, 2014.

2010 Mathematics Subject Classification: 13A02, 16W50.

Key words and phrases: graded quasi-prime submodules, graded prime submodules. 
by $g \in G$. If $x \in R$, then $x$ can be written uniquely as $\sum_{g \in G} x_{g}$, where $x_{g}$ is the component of $x$ in $R_{g}$. Moreover, $h(R)=\bigcup_{g \in G} R_{g}$. Let $I$ be an ideal of $R$. Then $I$ is called a graded ideal of $(R, G)$ if $I=\bigoplus_{g \in G}\left(I \cap R_{g}\right)$. Thus, if $x \in I$, then $x=\sum_{g \in G} x_{g}$ with $x_{g} \in I$. An ideal of a $G$-graded ring need not be $G$-graded (see Example 2.4 in [1]). Let $R=\bigoplus_{g \in G} R_{g}$ be a $G$-graded ring and let $I$ be a graded ideal of $R$. Then the quotient ring $R / I$ is also a $G$-graded ring. Indeed, $R / I=\bigoplus_{g \in G}(R / I)_{g}$ where $(R / I)_{g}=\left\{x+I: x \in R_{g}\right\}$. For the simplicity, we will denote the graded ring $(R, G)$ by $R$. Let $R$ be a $G$-graded $\operatorname{ring}$ and $M$ an $R$-module. We say that $M$ is a $G$-graded $R$-module (or graded $R$-module) if there exists a family of subgroups $\left\{M_{g}\right\}_{g \in G}$ of $M$ such that $M=\bigoplus_{g \in G} M_{g}$ (as abelian groups) and $R_{g} M_{h} \subseteq M_{g h}$ for all $g, h \in G$. Here, $R_{g} M_{h}$ denotes the additive subgroup of $M$ consisting of all finite sums of elements $r_{g} s_{h}$ with $r_{g} \in R_{g}$ and $s_{h} \in M_{h}$. Also, we write $h(M)=\bigcup_{g \in G} M_{g}$ and the elements of $h(M)$ are called homogeneous. Let $M=\bigoplus_{g \in G} M_{g}$ be a graded $R$-module and $N$ a submodule of $M$. Then $N$ is called a graded submodule of $M$ if $N=\bigoplus_{g \in G}\left(N \cap M_{g}\right)$. In this case, $N_{g}$ is called the $g$-component of $N$. Moreover, $M / N$ becomes a $G$-graded $R$-module with $g$-component $(M / N)_{g}=\left(M_{g}+N\right) / N$ for $g \in G$. Let $R$ be a $G$-graded ring and $S \subseteq h(R)$ be a multiplicatively closed subset of $R$. Then the ring of fraction $S^{-1} R$ is a graded ring which is called the graded ring of fractions. Indeed, $S^{-1} R=\bigoplus_{g \in G}\left(S^{-1} R\right)_{g}$ where $\left(S^{-1} R\right)_{g}=\{r / s: r \in R, s \in S$ and $\left.g=(\operatorname{deg} s)^{-1}(\operatorname{deg} r)\right\}$. Let $M$ be a graded module over a $G$-graded $\operatorname{ring} R$ and $S \subseteq h(R)$ be a multiplicatively closed subset of $R$. The module of fractions $S^{-1} M$ over a graded ring $S^{-1} R$ is a graded module which is called the module of fractions, if $S^{-1} M=\bigoplus_{g \in G}\left(S^{-1} M\right)_{g}$ where $\left(S^{-1} M\right)_{g}=\{m / s: m \in M, s \in S$ and $\left.g=(\operatorname{deg} s)^{-1}(\operatorname{deg} m)\right\}$. We write $h\left(S^{-1} R\right)=\cup_{g \in G}\left(S^{-1} R\right)_{g}$ and $h\left(S^{-1} M\right)=$ $\cup_{g \in G}\left(S^{-1} M\right)_{g}$. Consider the graded homomorphism $\eta: M \rightarrow S^{-1} M$ defined by $\eta(m)=m / 1$. For any graded submodule $N$ of $M$, the submodule of $S^{-1} M$ generated by $\eta(N)$ is denoted by $S^{-1} N$. Similar to non graded case, one can prove that $S^{-1} N=\left\{\beta \in S^{-1} M: \beta=m / s\right.$ for $m \in N$ and $\left.s \in S\right\}$ and that $S^{-1} N \neq S^{-1} M$ if and only if $S \cap\left(N::_{R} M\right)=\phi$. If $K$ is a graded submodule of an $S^{-1} R$-module $S^{-1} M$, then $K \cap M$ will denote the graded submodule $\eta^{-1}(K)$ of $M$. Moreover, similar to the non graded case one can prove that $S^{-1}(K \cap M)=K$. For more details, one can refer to [4].

\section{Some Properties of Graded Quasi-Prime Submodules}

In this section, we define the graded quasi-prime submodules and give some of their basic properties.

Definition 2.1. A proper graded submodule $N$ of a graded $R$-module $M$ is said to 
be a graded quasi-prime if whenever $K_{1}$ and $K_{2}$ are graded submodules of $M$ with $K_{1} \cap K_{2} \subseteq N$, either $K_{1} \subseteq N$ or $K_{2} \subseteq N$.

The following lemma is known, but we write it here for the sake of references.

Lemma 2.2.[3, Lemma 2.1] Let $R$ be a G-graded ring and $M$ a graded $R$-module. Then the following hold:

(i) If $I$ and $J$ are graded ideals of $R$, then $I+J$ and $I \cap J$ are graded ideals.

(ii) If $N$ is a graded submodule of $M, r \in h(R), x \in h(M)$ and $I$ is a graded ideal of $R$, then $R x, I N$ and $r N$ are graded submodules of $M$.

(iii) If $N$ and $K$ are graded submodules of $M$, then $N+K$ and $N \cap K$ are also graded submodules of $M$ and $\left(N:_{R} M\right)=\{r \in R: r M \subseteq N\}$ is a graded ideal of $R$.

(iv) Let $\left\{N_{\lambda}\right\}$ be a collection of graded submodules of $M$. Then $\sum_{\lambda} N_{\lambda}$ and $\bigcap_{\lambda} N_{\lambda}$ are graded submodules of $M$.

Recall that a proper graded submodule $N$ of a graded $R$-module $M$ is said to be graded irreducible if for each graded submodules $K_{1}$ and $K_{2}$ of $M, N=K_{1} \cap K_{2}$ implies that either $N=K_{1}$ or $N=K_{2}$.

Theorem 2.3. Let $R$ be a $G$-graded ring, $M$ a graded $R$-module and $N$ a graded submodule of $M$. If $N$ is a graded quasi-prime submodule of $M$, then $N$ is a graded irreducible submodule of $M$.

Proof. Assume that $N$ is a graded quasi-prime submodule of $M$ and $K_{1}, K_{2}$ are graded submodules of $M$ such that $N=K_{1} \cap K_{2}$. Since $N$ is a graded quasi-prime submodule and $K_{1} \cap K_{2} \subseteq N$, we have either $K_{1} \subseteq N$ or $K_{2} \subseteq N$ and hence either $N=K_{1}$ or $N=K_{2}$. Thus $N$ is a graded irreducible submodule.

Theorem 2.4. Let $R$ be a $G$-graded ring, $M$ a graded $R$-module and $N$ a graded quasi-prime submodule of $M$. If $V$ is a graded submodule contained in $N$, then $N / V$ is a graded quasi-prime submodule of $M / V$.

Proof. Let $K_{1}$ and $K_{2}$ be a graded submodules of $M$ such that $\left(K_{1} / V\right) \cap\left(K_{2} / V\right) \subseteq$ $N / V$. Then $K_{1} \cap K_{2}=\left(K_{1}+V\right) \cap\left(K_{2}+V\right) \subseteq N+V=N$. Since $N$ is a graded quasiprime submodule, either $K_{1} \subseteq N$ or $K_{2} \subseteq N$. It follows that either $K_{1} / V \subseteq N / V$ or $K_{2} / V \subseteq N / V$. Thus $N / V$ is a graded quasi-prime submodule.

In the following theorem, we give a characterization of graded quasi-prime submodules.

Theorem 2.5. Let $R$ be a $G$-graded ring, $M$ a graded $R$-module and $N$ a proper graded submodule of $M$. Then the following statements are equivalent.

(i) $N$ is a graded quasi-prime submodule of $M$. 
(ii) For every pair of elements $m, m^{\prime} \in h(M)$ such that $m R \cap m^{\prime} R \subseteq N$, either $m \in N$ or $m^{\prime} \in N$.

Proof. (i) $\Rightarrow$ (ii) This follows from Lemma 2.2(2) and the definition of graded quasi-prime.

(ii) $\Rightarrow$ (i) Let $K_{1}$ and $K_{2}$ be graded submodules of $M$ such that $K_{1} \cap K_{2} \subseteq N$ and $K_{1} \not \subseteq N$. Then there exists an element $k_{h} \in\left(K_{1} \cap h(M)\right)-N$. Now, let $g \in G$ and set $m=\sum_{g \in G} m_{g} \in K_{2}$. Then for all $g \in G, k_{h} R \cap m_{g} R \subseteq K_{1} \cap K_{2} \subseteq N$. By our assumption, we obtain $m_{g} \in N$. So $m \in N$, which indicates that $K_{2} \subseteq N$. Thus $N$ is a graded quasi-prime submodule of $M$.

Recall that a graded $R$-module $M$ is called graded multiplication if for each graded submodule $N$ of $M, N=I M$ for some graded ideal $I$ of $R$. One can easily show that if $N$ is a graded submodule of a graded multiplication module $M$, then $N=\left(N:_{R} M\right) M$, (see [5, Definition 2]). Also, a proper graded submodule $N$ of a graded $R$-module $M$ is called graded prime submodule if whenever $r \in h(R)$ and $m \in h(M)$ with $r m \in N$, either $r \in\left(N:_{R} M\right)$ or $m \in N$, (see [2, Definition $2.2]$ ). The following result provides some conditions under which a graded prime submodule is graded quasi-prime.

Theorem 2.6. Let $R$ be a $G$-graded ring, $M$ a graded multiplication $R$-module and $N$ a graded submodule of $M$. If $N$ is a graded prime submodule of $M$, then $N$ is a graded quasi-prime.

Proof. Assume that $N$ is a graded prime and let $K_{1}, K_{2}$ be graded submodules of $M$ such that $K_{1} \cap K_{2} \subseteq N$ but $K_{1} \nsubseteq N$ and $K_{2} \nsubseteq N$. Since $M$ is a graded multiplication, $K_{1}=J_{1} M$ and $K_{2}=J_{2} M$ for some graded ideals $J_{1}$ and $J_{2}$ of $R$. So there are $j_{1} \in J_{1} \cap h(R), j_{2} \in J_{2} \cap h(R)$ and $m_{1}, m_{2} \in h(M)$ such that $j_{1} m_{1} \notin N$ and $j_{2} m_{2} \notin N$. Since $N$ is a graded prime submodule and $j_{1} j_{2} m_{1} \in K_{1} \cap K_{2} \subseteq N$, we conclude that $j_{2} \in\left(N:_{R} M\right)$, i.e., $j_{2} M \subseteq N$. So $j_{2} m_{2} \in N$, a contradiction. Thus $N$ is graded quasi-prime.

Lemma 2.7. Let $R$ be a $G$-graded ring and $M$ a faithful graded multiplication R-module. Then $\bigcap_{\alpha \in \Delta}\left(I_{\alpha} M\right)=\left(\bigcap_{\alpha \in \Delta} I_{\alpha}\right) M$ where $I_{\alpha}$ is a graded ideal of $R$.

Proof. See [5, Theorem 8].

A proper graded ideal $P$ of a graded ring $R$ is said to be graded quasi-prime if for graded ideals $J_{1}$ and $J_{2}$ of $R$, the inclusion $J_{1} \cap J_{2} \subseteq P$ implies that either $J_{1} \subseteq P$ or $J_{2} \subseteq P$.

Theorem 2.8. Let $R$ be a G-graded ring, $M$ a faithful graded multiplication $R$ module and $N$ a graded submodule of $M$. Then $N$ is a graded quasi-prime submodule of $M$ if and only if $\left(N:_{R} M\right)$ is a graded quasi-prime ideal of $R$.

Proof. $(\Rightarrow)$ Assume that $N$ is a graded quasi-prime submodule. By Lemma 2.2(iii), $\left(N:_{R} M\right)$ is a graded ideal. Let $J_{1}$ and $J_{2}$ be graded ideals of $R$ such that $J_{1} \cap J_{2} \subseteq$ 
$\left(N:_{R} M\right)$, i.e., $\left(J_{1} \cap J_{2}\right) M \subseteq N$. By Lemma 2.7 , we have $\left(J_{1} \cap J_{2}\right) M=\left(J_{1} M\right) \cap$ $\left(J_{2} M\right) \subseteq N$. Since $N$ is a graded quasi-prime submodule of $M$, either $J_{1} M \subseteq N$ or $J_{2} M \subseteq N$ and so either $J_{1} \subseteq\left(N:_{R} M\right)$ or $J_{2} \subseteq\left(N:_{R} M\right)$. Thus $\left(N:_{R} M\right)$ is a graded quasi-prime ideal of $R$.

$(\Leftarrow)$ Assume that $\left(N:_{R} M\right)$ is a graded quasi-prime ideal of $R$ and let $K_{1}, K_{2}$ be graded submodules of $M$ such that $K_{1} \cap K_{2} \subseteq N$. Then $\left(K_{1} \cap K_{2}:_{R} M\right) \subseteq\left(N:_{R} M\right)$ and hence $\left(K_{1}:_{R} M\right) \cap\left(K_{2}:_{R} M\right) \subseteq\left(N:_{R} M\right)$. Since $\left(N:_{R} M\right)$ is a graded quasiprime ideal of $R$, either $\left(K_{1}:_{R} M\right) \subseteq\left(N:_{R} M\right)$ or $\left(K_{2}:_{R} M\right) \subseteq\left(N:_{R} M\right)$. Since $M$ is a graded multiplication, we conclude that either $K_{1}=\left(K_{1}:_{R} M\right) M \subseteq$ $\left(N:_{R} M\right) M=N$ or $K_{2}=\left(K_{2}:_{R} M\right) M \subseteq\left(N:_{R} M\right) M=N$. Thus $N$ is a graded quasi-prime submodule of $M$.

The graded radical of a graded ideal $I$, denoted by $G r(I)$, is the set of all $x=\sum_{g \in G} x_{g} \in R$ such that for each $g \in G$ there exists $n_{g}>0$ with $x_{g}^{n_{g}} \in I$. Note that if $r$ is a homogeneous element of $R$, then $r \in G r(I)$ if and only if $r^{n} \in I$ for some $n \in \mathbb{N}$, (see [7, Definition 2.1]). Recall that a proper graded ideal $P$ of $R$ is said to be a graded prime ideal if whenever $r, s \in h(R)$ with $r s \in P$, then either $r \in P$ or $s \in P$, (see [7].) The following theorem shows the relationship between graded prime submodules and graded quasi-prime submodules.

Theorem 2.9. Let $R$ be a $G$-graded ring, $M$ a faithful graded multiplication $R$ module and $N$ a graded submodule of $M$ such that $G r\left(\left(N:_{R} M\right)=\left(N:_{R} M\right)\right.$. Then $N$ is a graded quasi-prime submodule if and only if it is graded prime.

Proof. $(\Rightarrow)$ Assume that $N$ is a graded quasi-prime submodule. By Theorem 2.8, $\left(N:_{R} M\right)$ is a graded quasi-prime ideal of $R$. First, we show that $\left(N:_{R} M\right)$ is a graded prime ideal. Let $I_{1}, I_{2}$ be graded ideals of $R$ such that $I_{1} I_{2} \subseteq\left(N:_{R} M\right)$. Hence by [7, Proposition 2,4], we conclude that $I_{1} \cap I_{2} \subseteq G r\left(I_{1} \cap I_{2}\right) \subseteq G r\left(I_{1} I_{2}\right) \subseteq$ $\operatorname{Gr}\left(\left(N:_{R} M\right)\right)=\left(N:_{R} M\right)$. Since $\left(N:_{R} M\right)$ is a graded quasi-prime ideal, either $I_{1} \subseteq\left(N:_{R} M\right)$ or $I_{2} \subseteq\left(N:_{R} M\right)$. So $\left(N:_{R} M\right)$ is a graded prime ideal by [7, Proposition 1.2]. It follows that $N$ is a graded prime submodule of $M$ by [5, Corollary 3].

$(\Leftarrow)$ Theorem 2.6.

The following results study the behavior of graded quasi-prime submodules under localization.

Theorem 2.10. Let $N$ be a graded submodule of a graded $R$-module $M$ and $S \subseteq$ $h(R)$ be a multiplicatively closed subset of $R$. If $S^{-1} N$ is a graded quasi-prime submodule of $S^{-1} M$, then $S^{-1} N \cap M$ is a graded quasi-prime submodule of $M$.

Proof. Assume that $S^{-1} N$ is a graded quasi-prime submodule and let $K_{1}, K_{2}$ be graded submodules of $M$ such that $K_{1} \cap K_{2} \subseteq S^{-1} N \cap M$. It is easy to see that $S^{-1} K_{1} \cap S^{-1} K_{2} \subseteq S^{-1} N$. Since $S^{-1} N$ is a graded quasi-prime, either $S^{-1} K_{1} \subseteq$ $S^{-1} N$ or $S^{-1} K_{2} \subseteq S^{-1} N$ and hence either $K_{1} \subseteq S^{-1} N \cap M$ or $K_{2} \subseteq S^{-1} N \cap M$. Thus $S^{-1} N \cap M$ is a graded quasi-prime submodule. 
Recall that a proper graded submodule $N$ of a graded $R$-module $M$ is said to be a graded primary submodule if whenever $r \in h(R)$ and $m \in h(M)$ with $r m \in N$, then either $m \in N$ or $r \in G r\left(\left(N:_{R} M\right)\right)$ (see [5, Definition 6]).

Lemma 2.11. Let $N$ be a graded submodule of a graded $R$-module $M$ and $S \subseteq h(R)$ be a multiplicatively closed subset of $R$ such that $\operatorname{Gr}\left(\left(N:_{R} M\right)\right) \cap S=\phi$. If $N$ is a graded primary submodule of $M$, then $S^{-1} N \cap M=N$.

Proof. Let $x=\sum_{g \in G} x_{g} \in S^{-1} N \cap M$. Then for all $g \in G$, there are elements $n_{h} \in N \cap h(M)$ and $s \in S$ such that $\frac{x_{g}}{1}=\frac{n_{h}}{s}$. Hence there exists $t \in S$ such that $s t x_{g}=t n_{h} \in N$. Since $N$ is a graded primary submodule and $\operatorname{Gr}\left(\left(N:_{R} M\right)\right) \cap S=\phi$, $x_{g} \in N$. So $x \in N$, which shows that $S^{-1} N \cap M \subseteq N$. The opposite inclusion is obvious. Thus $S^{-1} N \cap M=N$.

Theorem 2.12. Let $N$ be a graded primary submodule of a graded $R$-module $M$ and $S \subseteq h(R)$ be a multiplicatively closed subset of $R$ such that $G r\left(\left(N:_{R} M\right)\right) \cap S=\phi$. If $N$ is a graded quasi-prime submodule of $M$, then $S^{-1} N$ is a graded quasi-prime submodule of $S^{-1} M$.

Proof. Assume that $N$ is a graded quasi-prime submodule of $M$ and let $K_{1}$, $K_{2}$ be graded submodules of $S^{-1} M$ such that $K_{1} \cap K_{2} \subseteq S^{-1} N$. Then $\left(K_{1} \cap\right.$ $M) \cap\left(K_{2} \cap M\right) \subseteq S^{-1} N \cap M$. By Lemma 2.11, $S^{-1} N \cap M=N$. Since $N$ is a graded quasi-prime submodule, either $K_{1} \cap M \subseteq N$ or $K_{2} \cap M \subseteq N$. So either $K_{1}=S^{-1}\left(K_{1} \cap M\right) \subseteq S^{-1} N$ or $K_{2}=S^{-1}\left(K_{2} \cap M\right) \subseteq S^{-1} N$. Thus $\bar{S}^{-1} N$ is graded quasi-prime.

\section{Topology on the Graded Quasi-Prime Submodules}

In this section, we introduce a topology on the set of graded quasi-prime submodules and some properties of this topology are given.

If $R$ is a $G$-graded ring and $M$ is a graded $R$-module, we consider $q \operatorname{Spec}_{g}(M)$ which is the set of all graded quasi-prime submodules of $M$. We call $q \operatorname{Spec}_{g}(M)$, the graded quasi-prime spectrum of $M$. For each subset $A \subseteq h(M)$, let $q V_{g}(A)=$ $\left\{P \in q \operatorname{Spec}_{g}(M): A \subseteq P\right\}$.

Theorem 3.1. Let $R$ be a G-graded ring and $M$ a graded $R$-module. Then the following hold:

(i) For each subset $A \subseteq h(M), q V_{g}(A)=q V_{g}(N)$, where $N$ is the graded submodule of $M$ generated by $A$.

(ii) $q V_{g}(0)=q \operatorname{Spec}_{g}(M)$ and $q V_{g}(M)=\phi$.

(iii) If $\left\{N_{\alpha}\right\}_{\alpha \in \Delta}$ is a family of graded submodules of $M$, then $\bigcap_{\alpha \in \Delta} q V_{g}\left(N_{\alpha}\right)=$ $q V_{g}\left(\sum_{\alpha \in \Delta} N_{\alpha}\right)$ 
(iv) For every pair $N$ and $K$ of graded submodules of $M, q V_{g}(N \cap K)=q V_{g}(N) \cup$ $q V_{g}(K)$.

Proof. (i) - (iii) Clear.

(iv) Let $N, K$ be any graded submodules of $M$ and $P \in q V_{g}(N \cap K)$. Then $N \cap K \subseteq P$. Since $P$ is a graded quasi-prime submodule, either $N \subseteq P$ or $K \subseteq P$, i.e., $P \in q V_{g}(N)$ or $P \in q V_{g}(K)$. Hence $q V_{g}(N \cap K) \subseteq q V_{g}(N) \cup q V_{g}(K)$. Other side of the inclusion is obvious. Thus $q V_{g}(N \cap K)=q V_{g}(N) \cup q V_{g}(K)$.

Let $q \zeta_{g}(M)=\left\{q V_{g}(N): N\right.$ is a graded submodule of $\left.M\right\}$. Then $q \zeta_{g}(M)$ contains the empty set and $q \operatorname{Spec}_{g}(M)$. Also, $q \zeta_{g}(M)$ is closed under arbitrary intersections and finite unions. Therefore, $q \zeta_{g}(M)$ satisfies the axioms for the closed sets of the unique topology $q \tau_{g}$ on $q \operatorname{Spec}_{g}(M)$. Then the topology $q \tau_{g}(M)$ on $q \operatorname{Spec}_{g}(M)$ is called the quasi-Zariski topology. Let $X=q \operatorname{Spec}_{g}(M)$. For every subset $S$ of $h(M)$, define $X_{S}=X-q V_{g}(S)$. In particular, if $S=\{a\}$, then we denote $X_{S}$ by $X_{a}$.

Theorem 3.2. Let $M$ be a graded $R$-module. Then the set $\left\{X_{a}: a \in h(M)\right\}$ is a basis for the quasi-Zariski topology on $X$.

Proof. Let $U$ be a non-void open subset of $X$. Then $U=X-q V_{g}(N)$ for some graded submodule $N$ of $M$. Assume that $N$ is generated by $A \subseteq h(M)$. Then $U=X-q V_{g}(N)=X-q V_{g}(A)=X-q V_{g}\left(\bigcup_{a \in A}\{a\}\right)=X-\bigcap_{a \in A} q V_{g}(a)=$ $\bigcup_{a \in A}\left(X-q V_{g}(a)\right)=\bigcup_{a \in A} X_{a}$.

For each graded submodule $N$ of a graded $R$-module $M$, we consider $q \operatorname{Gr}_{M}(N)=$ $\{P: P$ is a graded quasi-prime submodule of $M$ containing $N\}$.

Lemma 3.3. Let $N$ be a graded submodule of a graded $R$-module $M$. Then the following hold:

(i) $q V_{g}(N)=q V_{g}\left(q G r_{M}(N)\right)$.

(ii) For each graded submodule $K$ of $M, q V_{g}(K) \subseteq q V_{g}(N)$ if and only if $q G r_{M}(N) \subseteq q G r_{M}(K)$.

Proof. Clear

Recall that a topological space is said to be Noetherian if its closed sets satisfy the descending chain condition. Also, recall that a graded $R$-module $M$ is called graded Noetherian if it is satisfies the ascending chain condition on graded submodules of $M$.

Theorem 3.4. Let $R$ be a $G$-graded ring and $M$ a graded $R$-module. If $M$ is graded Noetherian, then $q_{S p e c}(M)$ is a Noetherian topological space.

Proof. Let $\cdots \subseteq q V_{g}\left(N_{3}\right) \subseteq q V_{g}\left(N_{2}\right) \subseteq q V_{g}\left(N_{1}\right)$ be a descending chain of closed subsets of $q \operatorname{Spec}_{g}(M)$, where $\left\{N_{k}\right\}_{k=1}^{\infty}$ is a family of graded submodules of $M$. 
By Lemma 3.3, we have $q G r_{M}\left(N_{1}\right) \subseteq q G r_{M}\left(N_{2}\right) \subseteq q G r_{M}\left(N_{3}\right) \subseteq \cdots$. Since $M$ is graded Noetherian, there exists a positive integer $k$ such that $q G r_{M}\left(N_{k}\right)=$ $q G r_{M}\left(N_{k+i}\right)$ for each $i=1,2,3, \ldots$ By Lemma 3.3, we conclude that

$$
q V_{g}\left(N_{k}\right)=q V_{g}\left(q G r_{M}\left(N_{k}\right)\right)=q V_{g}\left(q G r_{M}\left(N_{k+i}\right)\right)=q V_{g}\left(N_{k+i}\right)
$$

for all $i=1,2,3 \ldots$ Thus $q \operatorname{Spec}_{g}(M)$ is a Noetherian topological space.

Acknowledgments. The authors wish to thank sincerely the referees for their valuable comments and suggestions.

\section{References}

[1] R. Abu-Dawwas and M. Ali, Comultiplication modules over strongly graded rings, Int. J. Pure Appl. Math., 81(5)(2012), 693-699.

[2] S. E. Atani, On graded prime submodules, Chiang Mai J. Sci., 33(1)(2006), 3-7.

[3] F. Farzalipour and P. Ghiasvand, On the union of graded prime submodules, Thai. J. Math., 9(1)(2011), 49-55.

[4] C. Nastasescu and F. Van Oystaeyen, Graded Ring Theory, Mathematical Library 28, North Holand, Amsterdam, 1982.

[5] K. H. Oral, U. Tekir and A. G. Agargun, On graded prime and primary submodules, Turk. J. Math., 35(2011), 159-167.

[6] M. Refai and K. Al-Zoubi, On graded primary ideals, Turk. J. Math., 28(2004), 217229.

[7] M. Refai, M. Hailat, and S. Obiedat, Graded radicals and graded prime spectra, Far East J. Math. Sci. (FJMS), Part I, (2000), 59-73. 